\title{
Seleção e Análise de Estímulos na Tarefa de Busca Visual
}

\author{
Stimuli Selection and Analysis in the Visual Search Task
}

\author{
Joaquim Carlos Rossini“ ${ }^{a}$ \& Cesar Galera ${ }^{b}$ \\ ${ }^{a}$ Universidade Federal de Uberlândia, Uberlândia, Brasil, ${ }^{b}$ Universidade de São Paulo, Ribeirão Preto, Brasil
}

\begin{abstract}
Resumo
A capacidade de processar simultaneamente vários estágios cognitivos ainda é motivo de discordância entre os modelos que tentam compreender o processamento da informação visual. Muitos resultados experimentais apontam basicamente duas maneiras possíveis de fluxo da informação entre estágios de processamento: uma discreta e outra contínua. No presente estudo, dois experimentos foram delineados para investigar se a informação inicialmente descartada, em um estágio pré-atentivo, pode influenciar o processamento atentivo da informação selecionada em uma tarefa de busca visual. De maneira geral, os resultados observados nestes experimentos mostram que os estímulos inicialmente descartados em um processo automático de seleção não interferem no processamento atentivo dos estímulos na tarefa de busca visual. Este resultado apóia os modelos que predizem uma seleção precoce dos elementos relevantes para o processamento atentivo e uma transmissão discreta da informação entre os estágios cognitivos de processamento.

Palavras-chave. Busca visual; estágios de processamento; processamento discreto; processamento contínuo.
\end{abstract}

\begin{abstract}
The capacity of processing several cognitive stages simultaneously still generates a considerable disagreement among models that try to understand the processing of visual information. Many experimental results suggest two main possibilities for the information flow between information processing stages: a discrete or a continuous flow. Two experiments have in the present study to investigate were designed attentional processing whether the information at first discarded in a pre-attentive state can influence the of selected information in a visual search task. The results show that the stimuli initially discarded in an automatic process of selection do not interfere in the attention all processing of stimuli in a visual search. The results support the models that predict an early selection of the relevant stimuli and a discrete transmission of the information between the cognitive processing stages.

Keywords: Visual search; processing stages; discrete processing; continuous processing.
\end{abstract}

A todo o momento temos que selecionar objetos importantes ao nosso redor que, na maioria das vezes, estão circundados por vários objetos irrelevantes para o comportamento adaptativo. Isso fica claro quando guiamos nosso carro por uma estrada movimentada ou procuramos algum objeto importante na bagunça da nossa mesa de trabalho e, invariavelmente, descartamos objetos irrelevantes para focalizar a atenção no objeto alvo. Esta tarefa é relativamente fácil quando o objeto que procuramos é definido por uma característica única (ex. um lápis vermelho apresentado em meio a uma dúzia de lápis azuis) e consideravelmente mais difícil quando o alvo é definido por um conjunto de características (ex. encontrar um amigo em meio a vários rostos na multidão).

De acordo com a Teoria da Integração das Características (TIC) (Treisman \& Gelade, 1980; Treisman \& Sato,

\footnotetext{
"Endereço para correspondência: Universidade Federal de Uberlândia, Instituto de Psicologia, Campus Umuarama, Av. Pará, 1720, Bloco 2C, sala 2C-42, Uberlândia, MG, 38400-902. Tel./ Fax: (034) 3218 2296. E-mail: jrossini@fapsi.ufu.br

Trabalho derivado da dissertação de mestrado de Joaquim Carlos Rossini.

Apoio financeiro: Coordenação de Aperfeiçoamento de Pessoal de Nível Superior (CAPES)
}

1990), o processo de busca visual pode ocorrer basicamente de duas maneiras: sem a mobilização de recursos atentivos, portanto de maneira pré-atentiva, ou mediante a mobilização serial do foco da atenção. A busca préatentiva ocorre simultaneamente sobre todo o campo visual e permite a localização automática de um alvo definido por uma característica única no campo de busca. Para definir este processo, muitas vezes a literatura utiliza indiscriminadamente os termos processamento préatentivo, processamento automático, processos precoces e processos sensoriais, como sinônimos de um mesmo processo de decodificação mental. No entanto, todos estes termos buscam caracterizar processos rápidos de codificação que não mobilizam recursos cognitivos superiores para a seleção da informação visual relevante contida no ambiente.

Por outro lado, se o alvo é composto por uma combinação de várias características, a sua codificação torna-se consideravelmente mais lenta. Este processo lento de análise da informação é atribuído a uma estratégia serial de integração e identificação dos objetos representados na cena de busca. Para Treisman e Gelade (1980) e Treisman e Sato (1990), a atenção é o processo responsável pela integração das características de um objeto composto por 
vários atributos em uma posição específica no espaço retinotópico. Desta maneira, as posições ocupadas pelos objetos são integradas uma após a outra, em um processamento serial aleatório que se estende até a detecção do alvo. Este processo de busca por um conjunto de características é bem representado por um modelo de processamento em que o tempo de reação (TR) varia de forma linear em função do número de estímulos a serem analisados. Em condições em que o alvo está ausente, todos os estímulos relevantes são integrados e descartados através de um processo serial. Em condições em que o alvo está presente, em média, apenas a metade dos estímulos relevantes é integrada até a detecção do alvo, e o coeficiente angular da função TR é a metade do coeficiente angular observado nas condições em que o alvo esta ausente. Assim, a relação entre o tempo necessário para a detecção do alvo nas provas com o alvo presente e o número de elementos relevantes é expressa na função: TR $=\alpha+(\beta / 2)$ $(\mathrm{n}+1)$, onde, $\mathrm{n}$ é o número de objetos a serem integrados, Beta $(\beta)$ é o coeficiente angular da função TR relacionado diretamente ao tempo necessário para a integração e análise de cada item, e Alfa $(\alpha)$ é o tempo necessário para os demais processos relacionados à busca do objeto, tais como a representação inicial dos estímulos no sistema visual e a execução da resposta motora (Dosher, 1998).

O modelo de busca serial aleatória proposto inicialmente por Treisman e Gelade (1980) foi, no entanto, rapidamente contestado em vários estudos que mostraram que a análise atentiva é precedida de um processo de seleção grupal. Em um destes primeiros estudos, Egeth, Virzi e Garbart (1984) apresentaram evidências de que o processo de busca seria restrito apenas a um grupo de estímulos selecionados com base em uma característica em comum com o alvo. Por exemplo, se o alvo é uma barra vermelha orientada verticalmente e os distratores são barras verticais verdes e barras horizontais vermelhas, a busca se restringe apenas aos estímulos vermelhos. Através desse processo de seleção, os elementos não selecionados, ou irrelevantes para a busca, seriam descartados de maneira rápida e simultânea, como um objeto único de textura. Nessa situação o tempo necessário para a detecção do alvo é determinado basicamente pelo número de distratores vermelhos. Estes resultados foram corroborados no estudo realizado por Kaptein, Theewes e van der Hejden (1995), que manipularam, de forma independente, o número de estímulos a serem atendidos e não atendidos. Os resultados, assim como os de Egeth et al., mostram que o TR apresentou um aumento em função do número de estímulos a serem atendidos, mas não em função dos estímulos não atendidos. Estes resultados são tomados como evidência de que a seleção envolve inicialmente a segregação do campo em dois grupos de estímulos, um dos quais será analisado de forma serial até que o alvo seja encontrado.

Evidências de que o processo de busca é restrito a um subconjunto de estímulos foram obtidas em situações em que o número de estímulos a serem atendidos variou de maneira independente do número de estímulos a serem descartados (Kaptein et al., 1995; Nakayama \& Silverman,
1986; Poisson \& Wilkinson, 1992; Theeuwes, 1996; Zohary \& Hochstein, 1989). Essa estratégia mostra, de fato, que o TR varia em função do número de estímulos a serem atendidos, sem ser afetado pelo número de estímulos a serem ignorados, mas não informa muito sobre o processo de seleção. Alguns modelos de busca consideram que esse primeiro estágio seria equivalente a um processo de agrupamento pré-atentivo, que ocorreria simultaneamente por todo o campo de busca (Duncan \& Humphreys, 1989), mas que também poderia ser dirigido de maneira recursiva a regiões mais específicas do campo (Grossberg, Mingolla \& Ross, 1994; Humphreys \& Muller, 1993). No entanto, existem poucas evidências sobre a natureza do processo de seleção e sobre a relação entre a seleção e o processo de análise.

Vários estudos comparam o desempenho na tarefa de busca visual em situações nas quais os estímulos relevantes (o alvo e os distratores) são apresentados em um campo visual limpo, com o desempenho em situações mais realistas na qual o campo de busca é preenchido por elementos de fundo, dispostos como uma textura, e irrelevantes para a tarefa de busca. Esses resultados têm mostrado que a eficácia da busca visual não é prejudicada pela presença da textura, desde que esta seja suficientemente distinta dos estímulos relevantes em, pelo menos, uma característica visual importante, tal como a luminância (Galera, 1997, 2001, 2003; Galera, Lopes \& von Grünau, 2000). Nesse caso, a presença da textura não altera a taxa de busca $(\beta)$ da função TR, permitindo supor que a textura foi eliminada do processo de análise. No entanto, alguns resultados sugerem que este processo de seleção pode não ser realizado de maneira pré-atentiva ou com recursos ilimitados, pois o descarte da textura é acompanhado de um custo temporal de aproximadamente $40 \mathrm{~ms}$ expresso no termo constante da função TR $(\alpha)$. Além disso, a presença da textura tem um efeito menor sobre o TR nas provas com alvo do que nas provas sem alvo (Galera \& Eyk, 2000). Tomados em conjunto, estes resultados podem representar uma limitação para os modelos de seleção através de processos discretos, nos quais a seleção dos estímulos relevantes seria finalizada antes do início do processo de análise. Assim, uma possível interação entre a presença do alvo e da textura poderia sugerir que os processos de seleção e análise são temporalmente sobrepostos, e que a análise dos estímulos relevantes pode começar antes que o processo de seleção tenha sido finalizado.

O principal objetivo do presente estudo foi caracterizar a transmissão da informação entre os processos de seleção e análise na tarefa de busca visual. De maneira mais específica, procuramos determinar se os processos de seleção e de análise podem ser considerados discretos, ou seja, se a informação relevante é selecionada e, só então, analisada (Sternberg, 1998; Treisman \& Gelade, 1980; Treisman \& Sato, 1990) ou se o processo de seleção e de análise pode ser compreendido como um processo contínuo de fluxo da informação entre estágios de processamento (Eriksen \& Schultz, 1979; McClelland, 1979). De acordo com a lógica dos fatores aditivos (Sternberg, 1969; veja também Egeth \& Dagenbach, 1991; Pashler \& Badgio, 
1985), os fatores experimentais que influenciam estágios de processamento discretos e independentes têm efeitos aditivos sobre o TR, enquanto que fatores que atuam sobre um mesmo estágio apresentam efeitos interativos sobre o TR.

Nesta investigação foram considerados três fatores experimentais: o número de estímulos relevantes para a tarefa de busca visual (alvo e distratores); a similaridade entre alvo e distratores, e o contraste da luminância entre os estímulos relevantes e a textura. De acordo com o modelo linear, o TR será afetado de maneira superaditiva pela interação entre o número de estímulos a serem examinados e a similaridade entre o alvo e os distratores, pois ambos são processados em um mesmo estágio de análise atentiva da informação. Esta interação superaditiva será evidenciada por um coeficiente angular maior nas situações de similaridade alta do que nas situações de similaridade baixa. Estes dois fatores, número de estímulos e similaridade, devem apresentar um efeito temporal independente do efeito temporal atribuído ao fator contraste da luminância, que afeta o estágio de seleção da informação, caracterizando assim um fluxo discreto da informação.

No Experimento 1, dezessete níveis de contraste da luminância entre elementos relevantes e textura foram investigados. Destes, dois níveis de contraste foram utilizados no Experimento 2 que combinou de forma fatorial o número de elementos relevantes apresentados e o nível de similaridade entre elementos relevantes e alvo.

De maneira geral, o resultado do Experimento 1 sugere que a seleção dos estímulos relevantes atinge seu nível mais eficiente quando o contraste da luminância entre estímulos relevantes e textura é maior do que 60-70 \%. $\mathrm{O}$ resultado do segundo experimento mostrou que, apesar do descarte da textura representar um custo temporal relacionado à dificuldade de seleção, este custo só afeta a análise dos estímulos relevantes quando o contraste não permite uma seleção eficiente. Tal resultado apóia a idéia de que o processo de seleção é flexível e pode permitir um fluxo discreto da informação entre o estágio de seleção e de análise, quando os estímulos relevantes e os irrelevantes para a busca são suficientemente distintos.

\section{Experimento 1}

O contraste da luminância entre os estímulos relevantes e os elementos irrelevantes em uma tarefa de busca visual é apontado como um aspecto importante e precoce do processo de seleção da informação visual (Dosher, 1998; Theeuwes \& Kooi, 1994). Este experimento investigou o efeito temporal na seleção dos estímulos relevantes em 17 níveis de contraste da luminância entre elementos relevantes e textura em uma tarefa de busca visual.

\section{Método}

\section{Participantes}

Este experimento contou com a participação voluntária de sete sujeitos, quatro do sexo masculino e três do sexo feminino, com idades entre 18 e 48 anos (idade média de 25 anos e cinco meses e desvio-padrão $=10,27)$. Todos os participantes eram destros e apresentavam visão normal ou corrigida. O presente estudo foi aprovado pelo Comitê de Ética em Pesquisa, CEP- FFCLRP - 024/2001.

\section{Material}

A apresentação dos estímulos em um monitor NEC FE 950 com tela de 19 polegadas e o registro do tempo de reação dos participantes foram realizados pelo software E-prime 1.o (Schneider, Eschman \& Zuccoloto, 2002), e o controle da luminância dos segmentos dos estímulos foi feita com o fotômetro Tektronix, modelo J 17 LumaColor com cabeça de leitura J1803.

\section{Estímulos}

Os estímulos relevantes para a tarefa de busca visual (6 distratores e o alvo), eram sinais de soma $(+)$ formados pela conjunção de dois segmentos de 1 x 0,1 graus de ângulo visual apresentado com uma luminância constante de $65 \mathrm{~cd} / \mathrm{m}^{2}$ sobre o fundo escuro da tela $\left(\mathrm{O}, \mathrm{O} \mathrm{cd} / \mathrm{m}^{2}\right)$. O alvo apresentava o segmento vertical deslocado 0,18 graus de ângulo visual para a direita ou para a esquerda em relação ao ponto central do segmento horizontal. Os elementos da textura eram definidos pela conjunção de segmentos de 1,4 x 0,1 graus de ângulo visual, dispostos em um ângulo de $45^{\circ}(\times)$. Os estímulos relevantes foram distribuídos aleatoriamente em uma matriz imaginária com 49 posições ( $7 \times 7$ ), que cobria uma área de 8,7 por $8,7 \mathrm{~cm}^{2}$ no centro da tela do monitor. A distância entre o centro de duas caselas adjacentes era de 1,3 grau de ângulo visual. As posições da matriz não ocupadas pelos distratores e pelo alvo, eram ocupadas pelos elementos da textura. A casela central do arranjo era sempre ocupada pelo sinal de fixação composto por dois segmentos de $0,5 \times$ x 0,1 graus de ângulo visual e luminância constante de $65 \mathrm{~cd} / \mathrm{m}^{2}$, como representado na Figura $1 \mathrm{~A}$. Os elementos da textura foram apresentados com os seguintes níveis de luminância $\mathrm{em} \mathrm{cd} / \mathrm{m}^{2}: 2,5 ; 3,5 ; 6,5 ; 7,0 ; 8,5 ; 11,0 ; 13,5 ; 17,0 ; 21,0 ; 25,5$; 29,$0 ; 33,5 ; 38,5 ; 43,5 ; 48,5 ; 55,0 ; 61$, O. O contraste entre os elementos da textura e os estímulos relevantes foi calculado através da fórmula de Michelson $\mathrm{C}=\left(\mathrm{L}_{\mathrm{r}}-\mathrm{L}_{\mathrm{t}} / \mathrm{L}_{\mathrm{r}}+\right.$ $\mathrm{L}_{\mathrm{t}}$ ), onde: $\mathrm{C}$ expressa o contraste da luminância dos estímulos, $\mathrm{L}_{\mathrm{r}}=$ luminância dos estímulos relevantes e $\mathrm{L}_{\mathrm{t}}=$ luminância dos elementos da textura, e foram os seguintes: 0,92; O,88; O,81; 0,80; O,76; O,70; 0,65; 0,57; 0,50; 0,43; 0,38; 0,31; 0,25; 0,19; 0,14; 0,08; 0,03.

\section{Procedimento}

A tarefa de busca visual exigiu que o participante discriminasse se havia um deslocamento do segmento vertical do alvo para a direita ou para a esquerda. Os 17 níveis de contraste da luminância foram apresentados de maneira aleatória e todas as provas contavam com a presença do alvo. Em metade das provas o segmento apresentava um deslocamento para a direita, e na outra metade para a esquerda. Cada prova era iniciada com a apresentação de um sinal de fixação “+” com $0,5^{\circ}$ x 0,5 $5^{\circ}$ de ângulo visual, que 
permanecia no centro da tela até a conclusão da prova. Após 1s de apresentação do sinal de fixação, todos os estímulos eram apresentados simultaneamente e permaneciam na tela até a resposta do participante. Imediatamente após a resposta do sujeito, uma mensagem de "resposta correta" ou "incorreta" (feedback) era apresentada por 1s, juntamente com o tempo gasto para a resposta ao alvo, encerrando a prova. A Figura 1 B mostra a seqüência de eventos apresentados em cada prova do experimento.

A

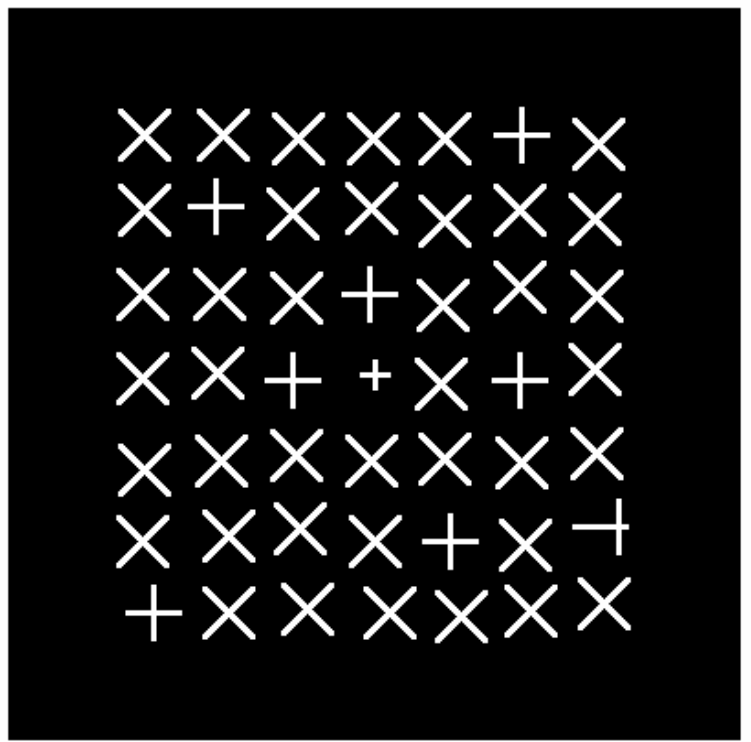

B

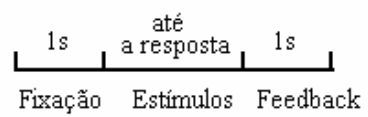

Figura 1. Representação esquemática dos estímulos apresentados no Experimento 1 e 2 . A textura era apresentada com uma luminância menor que os estímulos relevantes. B. Seqüência de eventos apresentados em cada prova

A tarefa do participante era pressionar a tecla "1" do teclado numérico caso o alvo apresentasse um deslocamento do segmento vertical para a esquerda, ou a tecla " 2 " do teclado numérico caso o alvo apresentasse um deslocamento do segmento vertical para a direita. O participante era instruído a ser o mais rápido possível, sem cometer erros. O ambiente experimental era reservado somente a tarefa, e o monitor era a única fonte luminosa do recinto. Os participantes permaneceram sentados, com os olhos a aproximadamente $57 \mathrm{~cm}$ de distância do monitor, com os dedos indicador e médio da mão direita sobre as teclas " 1 " e " 2 " do teclado numérico.

Cada sujeito participou de uma sessão experimental composta por dois blocos de 170 provas (20 provas por nível de contraste). Antes do primeiro bloco de provas os participantes realizavam 20 provas treino desconsideradas na análise dos dados.

\section{Resultados e Discussão}

O tempo de reação médio (TR) de cada participante, obtido em cada um dos 17 níveis de contraste foi submetido a uma análise da variância para medidas repetidas (ANOVA). O tamanho do efeito atribuído à variável independente foi estimado através do cálculo do Eta quadrado parcial $\left(\eta^{2}\right)$ que descreve a proporção da variabilidade total atribuída ao fator investigado. Os tempos de reação que excederam dois desvios padrão além da média do TR obtido por cada participante, em cada condição experimental, não foram incluídos na análise dos dados. Esta análise confirmou uma diminuição significativa do TR com o aumento do nível de contraste entre os estímulos relevantes e a textura $\left(F(16,96)=7,03 ; p<0,01 ; \eta^{2}=0,54\right)$. O tempo de reação dos participantes em função do contraste da luminância e mostrado na Figura 2.

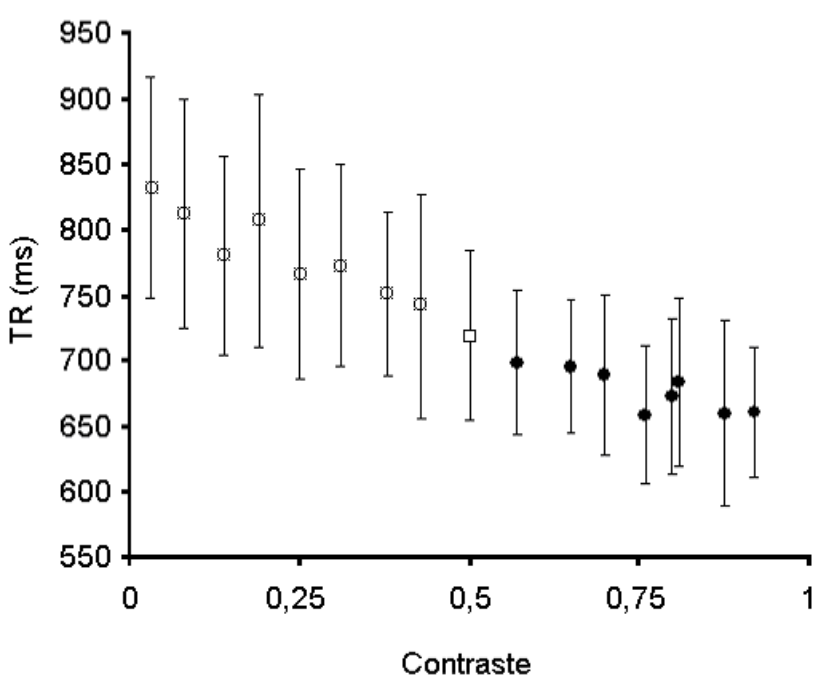

Figura 2. Tempo de reação dos participantes em função do contraste da luminância entre os estímulos relevantes e a textura. A medida de dispersão representa o erro-padrão em cada condição. (•) TR médio com contraste superior à $0,5=677 \mathrm{~ms}$; $(\square)$ TR médio com contraste igual à $0,5=$ $719 \mathrm{~ms}$; (o) TR médio com contraste inferior à $0,5=783 \mathrm{~ms}$.

O TR diminui até um ponto assintótico no qual o contraste era de $75 \%$. Análises exploratórias feitas através do teste post hoc de Newman-Keuls para identificação de grupos homogêneos (Alfa $=0,05)$ permitiram identificar dois grupos de contraste com sobreposição mínima em torno do contraste com valor de 50\%. Uma segunda análise realizada em separado confirmou que os TRs obtidos com os contrastes acima de 0,50 não apresentaram uma diferença significativa entre si $(\mathrm{TR}=677 \mathrm{~ms})(F(7,42)=1,45 ; p \geq$ 0,20). A mesma análise foi realizada com os contrastes inferiores a 0,50 e também confirmou que não existem diferenças significativas nos tempos de reação entre estes contrastes $(\mathrm{TR}=783 \mathrm{~ms})(F(7,42)=2,00 ; p \geq 0,07) . \mathrm{A}$ análise dos TRs com o teste post-hoc de Newman-Keuls $($ Alfa $=0,05)$ revelou uma diferença significativa entre estes dois grupos de $105 \mathrm{~ms}$. O percentual de erros foi inferior a $5 \%$ e não foi analisado. 
O Experimento 1 sugere a existência de um custo temporal associado à seleção da informação relevante apresentada em meio a um padrão de textura. Os resultados mostram dois momentos no processo de seleção da informação relevante. Em um primeiro momento, quando o contraste da luminância era inferior a 50\%, o custo temporal médio da busca visual foi significativamente maior do que quando o contraste da luminância era superior a 50\% (783 ms e $677 \mathrm{~ms}$, respectivamente). Nagy e Sanchez (1992) também encontraram uma diminuição do TR em função do aumento no contraste entre o alvo e distratores na tarefa de busca visual. De acordo com esses autores, a diminuição do TR até um valor assintótico sugere a existência de dois estágios de busca, um serial e um paralelo. Nossos resultados sugerem que um processo semelhante parece ocorrer na seleção dos estímulos relevantes. Dessa forma, selecionamos para o segundo experimento um nível de contraste que poderia caracterizar um processo de seleção serial (25\%) e um valor que permitiria um processo de seleção em paralelo (75\%).

\section{Experimento 2}

De acordo com o modelo linear da integração das características os dois parâmetros livres da função que relaciona o TR ao número de estímulos, o coeficiente angular $(\beta)$ e o intercepto da função $(\alpha)$, representam, respectivamente, o tempo necessário para a análise de cada estímulo relevante e o tempo associado aos processos não atencionais, tais como o de seleção (Dosher, 1998). Na hipótese de um fluxo discreto da informação, a seleção dos estímulos relevantes não deve reverter em um custo temporal vinculado ao coeficiente angular da função TR. Por outro lado, um fluxo contínuo da informação poderia ser evidenciado por uma interação subaditiva entre os fatores número de estímulos relevantes e a presença da textura.

Neste experimento foram manipulados três fatores: a similaridade entre o alvo e os distratores, o número de estímulos relevantes (alvo e distratores), e o contraste da luminância entre os estímulos relevantes e a textura. De acordo com a lógica dos fatores aditivos, uma interação entre os fatores similaridade e número de elementos relevantes aliada a uma independência do fator contraste da luminância, pode ser considerada uma indicativa de um fluxo discreto da informação entre os estágios de seleção e de análise da informação visual.

\section{Método}

\section{Participantes}

O Experimento 2 contou com a participação voluntária de doze sujeitos, nove do sexo feminino e três do sexo masculino, com idades entre 18 e 26 anos (idade média de 23 anos e dois meses e desvio padrão $=2,13$ ). Todos os participantes eram destros e apresentavam visão normal ou corrigida. O presente estudo foi aprovado pelo Comitê de Ética em Pesquisa, CEP- FFCLRP - 024/2001.

\section{Material}

O material utilizado para a realização do Experimento 2 foi o mesmo utilizado no Experimento 1.

\section{Estímulos}

Os estímulos visuais (estímulos relevantes e a textura) foram os mesmos do experimento anterior. O estímulo alvo apresentava duas configurações possíveis: com o segmento vertical deslocado $0,17^{\circ}$ de ângulo visual para a direita ou para a esquerda em relação ao ponto central do segmento horizontal (similaridade alta), ou com o segmento vertical deslocado $0,39^{\circ}$ de ângulo visual para a direita ou para a esquerda em relação ao ponto central do segmento horizontal (similaridade baixa). A textura, quando presente, apresentava um contraste da luminância de $25 \%$ (contraste baixo) ou $75 \%$ (contraste alto) em relação aos estímulos relevantes. Em 33\% das provas a textura não foi apresentada e a cena de busca contava apenas com os estímulos relevantes (sem textura).

\section{Procedimento}

A tarefa experimental e o procedimento foram iguais aos utilizados no primeiro experimento. Os participantes realizaram 540 provas, nas quais foram apresentados 18 tratamentos experimentais derivados do arranjo dos três fatores manipulados: o número de estímulos relevantes (2, 4 e 8); o contraste da luminância entre estímulos relevantes e a textura nos seguintes níveis: baixo (25\%), al to (75\%), e uma situação sem textura, considerada como o terceiro nível deste fator; e fator similaridade entre o alvo e os distratores (alta, baixa). Cada participante passou por uma sessão experimental composta por dois blocos de 270 provas. Cada tratamento foi apresentado 30 vezes de maneira aleatória ao longo dos dois blocos de prova. Antes do primeiro bloco os participantes realizavam 20 provas treino, desconsideradas na análise dos dados.

\section{Resultados e Discussão}

A Figura 3 mostra o tempo de reação médio dos participantes em função das condições experimentais investigadas. Uma vez que nosso objetivo neste experimento era determinar a natureza dos processos de seleção em relação a uma situação sem elementos de fundo, optamos por comparar separadamente os resultados obtidos nas provas com contraste al to e baixo ao desempenho nas provas sem textura. Dessa forma, os resultados obtidos em cada nível de contraste foram comparados aos resultados obtidos nas provas sem textura através de uma análise de variância com medidas repetidas (ANOVA) nos seguintes fatores: número (de estímulos relevantes 2, 4, 8); similaridade entre alvo e distratores (alta ou baixa), e textura (presença ou ausência). Os tempos de reação que excederam dois desvios padrão além da média do TR obtido por cada participante em cada condição experimental não foram incluídos na análise dos dados.

De maneira geral, nas provas com contraste alto, o TR aumenta em função do número de estímulos relevantes a 
uma taxa de 7,4 ms/item $\left(F(2,22)=26,816 ; p<0,01 ; \eta^{2}=\right.$ $\mathrm{O}, 71)$. O TR nas provas com similaridade alta (média do $\mathrm{TR}=636 \mathrm{~ms}$ ) é $105 \mathrm{~ms}$ maior do que nas provas com similaridade baixa (média do $\mathrm{TR}=531 \mathrm{~ms})(F(1,11)=$ 234, 919, $\left.p<0,01 ; \eta^{2}=0,95\right)$. Nas provas com textura o TR é $25 \mathrm{~ms}$ mais lento do que nas provas sem textura (596 ms e $571 \mathrm{~ms}$, respectivamente) $(F(1,11)=56,756, p$ $\left.<0,01, \eta^{2}=0,84\right)$. O efeito da textura independe tanto do número $\left(F(2,22)=0,136 ; p=0,87 ; \eta^{2}=0,01\right)$ como da similaridade entre estímulos relevantes e o alvo $(F(1,11)$ $\left.=2,377 ; p=0,15 ; \eta^{2}=0,18\right)$. Esta análise das provas com contraste alto mostra, ainda, uma interação superaditiva entre a similaridade e o número de estímulos relevantes $\left(F(2,22)=10,22 ; p<0,01 ; \eta^{2}=0,48\right)$. Esta interação se manifesta de maneira clara no coeficiente angular que relaciona o TR ao número de estímulos. Os estímulos mais similares são analisados mais lentamente $(11 \mathrm{~ms} /$ item $)$ do que estímulos menos similares (4 ms/item). A interação tripla não significativa entre a presença da textura, a similaridade, e o número de estímulos relevantes $(F(2,22)=$
0,095; $\left.p=0,91 ; \eta^{2}=0,01\right)$ mostra que a interação entre número e similaridade não é afetada pela presença da textura. Tanto nas provas com textura como nas provas sem textura a taxa de análise dos estímulos relevantes com similaridade alta é de $10 \mathrm{~ms} /$ item. O mesmo acontece nas provas com similaridade baixa, neste caso a taxa de busca é de 4,3 ms/item, tanto na presença como na ausência da textura.

De acordo com o método dos fatores aditivos (Sternberg, 1969, 1998), a interação superaditiva entre número de estímulos relevantes e a similaridade sugere que estes fatores experimentais atuam sobre um mesmo estágio de processamento, teoricamente responsável pela análise dos estímulos relevantes. Ainda de acordo com esse método, nossos resultados sugerem que a presença da textura afetou um estágio diferente, independente daquele afetado pela similaridade e pelo número de estímulos relevantes. Nós consideramos que esse estágio é responsável pela seleção dos estímulos relevantes.

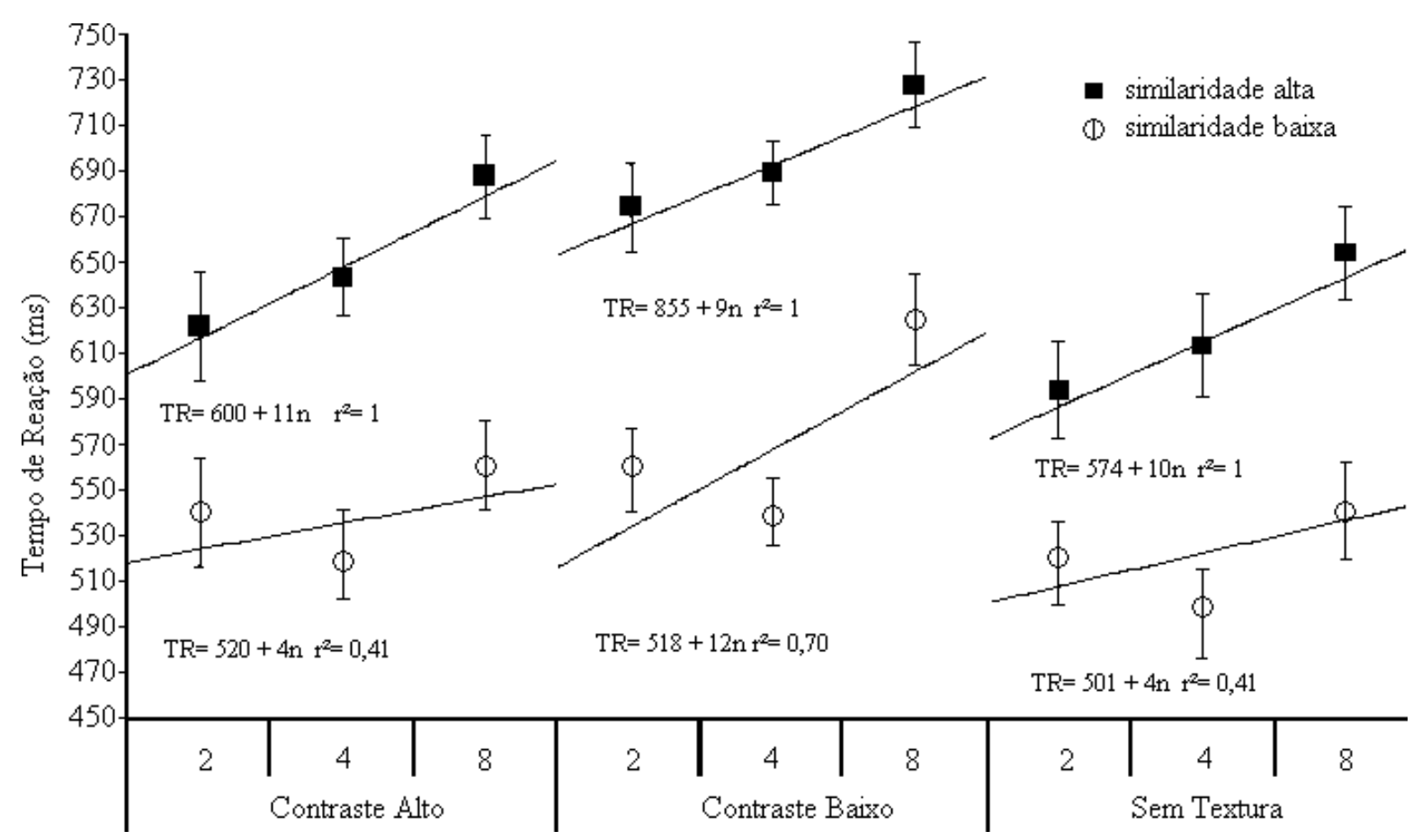

Figura 3. Experimento 2. Média do tempo de reação (TR) em função dos seguintes fatores: contraste da luminância (alto, baixo, sem textura); número de estímulos relevantes $(2,4,8)$; similaridade alvo / distratores (alta, baixa). A função TR e o quadrado do coeficiente de correlação são apresentados abaixo de cada regressão. A medida de dispersão representa o erro-padrão.

Na comparação entre o desempenho nas provas com textura de contraste baixo e nas provas sem textura o quadro de interações é mais complexo. Nesta condição o efeito principal do fator número de estímulos relevantes é significativo $\left(F(2,22)=52,364 ; p<0,01 ; \eta^{2}=0,83\right)$, com uma taxa de aumento do TR em função do número de estímulos de $9 \mathrm{~ms} /$ item. Há interação significativa entre os fatores similaridade e número de estímulos relevantes $(F(2$, $\left.22)=8,620, p<0,01, \eta^{2}=0,44\right)$ que sugere que a duração do processo de análise, estimada pelo coeficiente que relaciona o TR ao número de estímulos varia em função da similaridade. De maneira diferente do que acontece nas provas com textura de contraste alto, existe uma interação entre similaridade, número de estímulos relevantes e a presença da textura, na qual o efeito da similaridade é maior (122 ms, TR similaridade alta $=697 \mathrm{~ms}$, TR similaridade baixa $=575 \mathrm{~ms}$ ) nas provas em que a textura está presente do que nas provas em que estão presentes apenas os 
estímulos relevantes $(100 \mathrm{~ms}$, TR similaridade alta $=621$ ms, TR similaridade baixa $=521 \mathrm{~ms})(F(1,11)=7,911, p$ $\left.<0,02 ; \eta^{2}=0,42\right)$. No geral, a presença da textura provoca um aumento de $65 \mathrm{~ms}$ no TR (TR com textura $=636$ $\mathrm{ms}$, TR sem textura $=571 \mathrm{~ms})(F(1,11)=204,446, p<$ 0,$\left.01 ; \eta^{2}=0,95\right)$, e interage de maneira superaditiva com a similaridade. A interação entre o número de estímulos relevantes e a presença da textura é marginal $(F(2,22)=$ 3,305, $\left.p=0,056 ; \eta^{2}=0,23\right)$, mas contribui de forma significativa na interação tripla com a similaridade alvo-distrator $\left(F(2,22)=10,906, p<0,01 ; \eta^{2}=0,50\right)$. De acordo com essa interação a taxa de busca do alvo não é afetada pela presença da textura quando a similaridade alvo-distrator é alta.

Assim como na condição de contraste alto a interação superaditiva entre número de estímulos relevantes e a similaridade sugere que estes fatores experimentais atuam sobre um mesmo estágio de processamento, teoricamente responsável pela análise dos estímulos relevantes. Todavia, na condição de contraste baixo, a não interação entre o fator número e o fator textura é limítrofe e há uma interação significativa entre o fator similaridade e o fator textura. Isto sugere que, em condições em que o contraste entre os estímulos relevantes e os estímulos irrelevantes é pequeno, a eficiência do processo de descarte dos estímulos que compõem a textura diminui.

Os erros cometidos nas provas com contraste al to e baixo foram comparados separadamente ao desempenho nas provas sem a textura. A porcentagem de erros em cada condição foi submetida à mesma análise estatística utilizada para o tempo de reação. Nas provas com contraste alto houve um aumento significativo do erro vinculado ao número de estímulos relevantes com $5 \%, 7 \%$ e $10,8 \%$ para 2 , 4 e 8 estímulos respectivamente $(F(2,22)=5,17, p<0,02)$. Foram cometidos menos erros nas provas com similaridade baixa $(2,2 \%)$ do que com similaridade alta $(13 \%)$ $(F(1,11)=33,97, p<0,01)$. A interação entre número e similaridade também é significativa $(F(2,22)=8,57, p<0,01)$. A taxa de erro não foi alterada pela presença da textura $(7,9 \%)$ ou pela a sua ausência $(7,2 \%)(F(2,22)=0,78, p=$ o,39). A análise do erro nas condições de contraste baixo indicou um aumento significativo do erro vinculado aos três fatores investigados: número de elementos relevantes: $2(6,7 \%), 4(8,8 \%)$, e $8(15,2 \%)(F(2,22)=18,09, p<$ O,O1; similaridade, baixa $(3,1 \%)$, alta $(17,4 \%)(F(1,11)=$ $64,12, p<0,01)$; e contraste (textura ausente, $7,3 \%$ ), (textura presente, $13,2 \%)(F(1,11)=19,31, p<0,01)$. O fator número apresentou uma interação significativa com o fator similaridade $(F(2,22)=14,11, p<0,01)$ e com o fator contraste $(F(2,22)=5,47, p<0,02)$. Houve uma interação significativa entre o fator similaridade e o fator contraste $(F(1,11)=16,7, p<0,01)$. Uma análise separada da porcentagem de erros cometidos mostrou um aumento significativo do erro na condição com oito elementos relevantes, contraste baixo e similaridade baixa (6,8\%) em comparação a porcentagem de erros cometidos nas condições com oito elementos relevantes, sem a presença da textura, similaridade baixa $(2,2 \%)$ e oito elementos relevantes, contraste al to e similaridade baixa $(2,4 \%)(F(2,22)$ $=4,55, p<0,03)$.

\section{Discussão Geral}

Em linhas gerais, nossos resultados mostram que o descarte dos elementos da textura apresenta certo custo temporal (Experimento 1), e que este custo não está vinculado ao processo de análise (Experimento 2). Este resultado mostra que a presença dos elementos da textura não interfere no processo de busca, desde que a similaridade entre os estímulos relevantes seja grande e a similaridade entre estímulos relevantes e o elemento da textura seja pequeno (Galera, 2001; Galera et al., 2000).

Os resultados observados no segundo experimento sugerem que o fluxo da informação entre o estágio de seleção e o estágio de análise atentiva é discreto, uma vez que nas condições em que a similaridade entre alvo e distratores era grande, o coeficiente angular da função TR não foi afetado pela presença dos elementos da textura. Nesta condição, o custo temporal atribuído ao processo de descarte da textura está vinculado ao intercepto da função TR. De acordo com Dosher (1998), o custo temporal associado aos valores expressos no parâmetro intercepto da função TR não são atentivos. Todavia, nas condições em que a similaridade entre o alvo e os distratores era pequena, o coeficiente angular da função TR apresentou uma variação significativa na condição com oito elementos relevantes e $25 \%$ de contraste da luminância entre estímulos relevantes e textura. A análise do erro cometido nesta condição mostrou que este aumento do tempo de reação foi acompanhado de um aumento significativo da porcentagem de erros cometidos.

Alguns estudos sugerem que o sistema visual pode segmentar os estímulos presentes no campo em função das características que os definem, submetendo-os assim, a um processo de busca em paralelo, sem que o TR seja afetado pelo número de estímulos relevantes. Neste sentido, Nakayama e Silverman (1986) mostraram que o desempenho na tarefa de busca não é afetado pelo número de distratores quando o alvo é definido pela conjunção de disparidade estereoscópica e cor, ou disparidade estereoscópica e direção do movimento. Os autores sugerem que o sistema visual apresenta a capacidade de selecionar apenas os estímulos pertencentes ao plano de profundidade que contém o alvo, eliminando do processo de busca os estímulos contidos em outro plano. Nesta condição, a busca no plano selecionado torna-se uma busca restrita a uma característica única, que salta aos olhos dentre os estímulos selecionados, em uma estratégia de busca identificada por Treisman e Gelade (1980) como pop-out de segunda ordem. No Experimento 2 esta estratégia não foi eficiente na condição em que o número de elementos relevantes era elevado (oito elementos) e o contraste da luminância entre os estímulos relevantes e a textura era pequeno (25\%). Tal fato pode ter proporcionado um aumento na taxa de busca, expresso no coeficiente angular, para um valor próximo ao observado com elementos relevantes mais similares. No entanto, acreditamos que novos estudos serão necessários para esclarecer melhor este resultado.

De modo geral, o resultado da presente investigação corrobora vários resultados anteriores que mostram que, 
em algumas condições experimentais, a presença dos elementos de textura não interfere no processo de busca. Por exemplo, Galera et al. (2000) mostraram que quando os elementos da textura diferem dos estímulos relevantes na luminância dos seus componentes lineares, ou em aspectos mais gerais da forma, o tempo de reação dos participantes em uma tarefa de busca visual é afetado de maneira independente pelo número de estímulos relevantes e pela presença da textura. Neste caso a taxa de busca é a mesma, independente da presença da textura.

Neste estudo esta afirmação é sustentada pela constância do valor do coeficiente angular da função TR em todas as condições de grande similaridade entre o alvo e os distratores, o que indica um fluxo discreto da informação na busca visual nesta condição.

\section{Referências}

Dosher, B. A. (1998). Models of visual search: Finding a face in the crowd. In D. Scarborough \& S. Sternberg (Eds.), An invitation to Cognitive Science: Vol. 4. Methods, models, and conceptual issues (pp. 455-521). Cambridge, MA: MIT Press.

Duncan, J., \& Humphreys, G. W. (1989). Visual search and stimulus similarity. Psychological Review, 96, 433-458.

Egeth, H., \& Dagenbach, D. (1991). Parallel versus serial processing in visual search: Further evidence from subadditive effects of quality. Journal of Experimental Psycholoy: Human Perception \& Performance, 17, 55 1-560.

Egeth, H. E., Virzi, R. A., \& Garbart, H. (1984). Searching for conjunctively defined targets. Journal of Experimental Psychology: Human Perception and Performance, 10, 32-39.

Eriksen, E., \& Schultz, D. W. (1979). Information process in visual search: A continuous flow conception and experimental results. Perception \& Psychophysics, 25, 249-263.

Galera, C. (1997). Agrupamento por similaridade e busca visual. Psicologia: Teoria e Pesquisa, 13, 261-268.

Galera, C. (2001). O tamanho dos estímulos como critério de seleção na tarefa de busca visual. Psychologica, 28, 207-220.

Galera, C. (2003). Stimuli selection and attentive analysis in the visual search task: Evidences of discrete and sequential stages. Arquivos Brasileiros de Oftalmologia, 66, 5(Suppl.), 114-120.

Galera, C., \& Eyk, G. (2000). Target-distractor similarity affects the selection efficacy of the relevant stimuli in a modified visual search task [Abstract]. Annual Meeting 2000 of Association for Research in Vision and Oftalmology, 41(Suppl.), s424.

Galera, C., Lopes, E. J., \& von Grünau, M. (2000). Stimulus segmentation in the visual search task. Perception \& Psychophysics, 62, 505-516.

Grossberg, S., Mingolla, E, \& Ross, W. (1994). A neural theory of attentive visual search: Interaction of boundary, surface, spatial, and object representation. Psychological Reviere, 101, 470-489.

Humphreys, G., \& Müller, H. (1993). Search via recursive rejection (SERR): A connectionist model of visual search. Cognitive Psychology, 25, 43-110.

Kaptein, N. A., Theeuwes, J., \& van der Hejden, A. H. C. (1995). Search for a conjunctively defined target can be selectively limited to a colour-defined subset of elements. Journal of Experimental Psychology: Human Perception and Performance, 21, 1053-1069
McClelland, J. L. (1979). On the time relations of mental process: An examination of systems of processes in cascade. Psychological Review; 86, 287-330.

Nagy, A., \& Sanchez, R. R. (1992). Chromaticity and luminance as coding dimensions in visual search. Human Factors, 34, 601614 .

Nakayama, K., \& Silverman, G. H. (1986). Serial and parallel processing of visual feature conjunctions. Nature, 320, 264265.

Pashler, H., \& Badgio, P. C. (1985). Visual attention and stimulus identification. Journal of Experimental Psychology: Human Perception \& Performance, 11, 105-121.

Poisson, M. E., \& Wilkinson, F. (1992). Distractor ratio and grouping process in visual conjuction search. Perception, 21, 21-38.

Schneider, W., Eschman, A., \& Zuccoloto, A. (2002). E-prime 1.0 [Computer software]. Pittsburgh, PA: Psychological Software.

Sternberg, S. (1969). The discovery of processing stages: Extensions of donders' method. In W. G. Koster (Ed.), Attention and performance II (pp. 276-315). Amsterdam, NorthHolland: Elsevier.

Sternberg, S. (1998). Discovering mental processing stages: The method of additive factors. In D. Scarborough \& S. Sternberg (Eds.), An invitation to Cognitive Science: Vol. 4. Methods, models, and conceptual issues (pp. 703-863). Cambridge, MA: MIT Press.

Theeuwes, J. (1996). Parallel search for a conjunction of color and orientation: The effect of spatial proximity. Acta Psychologica, 94, 291-307.

Theeuwes, J., \& Kooi, F.L. (1994). Parallel search for conjunction of shape and contrast polarity. Vision Research, 34, 3013-3016.

Treisman, A., \& Gelade, G. (1980). A feature integration theory of attention. Cognitive Psychology, 12, 97-136.

Treisman, A., \& Sato, S. (1990). Conjunction search revisited. Journal of Experimental Psychology: Human Perception and Performance, 16, 459-478.

Zohary, E., \& Hochstein, S. (1989). How serial is serial processing in vision? Perception, 18, 191-20.
Recebido: 11/07/2006 $I^{a}$ revisão: 27/10/2006 $2^{a}$ revisão: $23 / 03 / 2007$ Aceite final: 21/05/2007 